\title{
The Fine Intersection Problem for Steiner Triple Systems
}

\author{
Yeow Meng Chee ${ }^{1,2}$, Alan C. H. Ling ${ }^{3}$, Hao Shen ${ }^{4}$ \\ 1 Division of Mathematical Sciences, School of Physical and Mathematical Sciences, \\ Nanyang Technological University, Singapore 637616, e-mail: ymchee@ntu.edu.sg. \\ 2 Card View Pte. Ltd., 41 Science Park Road, \#04-08A The Gemini, Singapore Science \\ Park II, Singapore 117610, e-mail: ymchee@alumni.uwaterloo.ca \\ 3 Department of Computer Science, University of Vermont, Burlington, Vermont 05405. \\ USA, e-mail: aling@emba.uvm.edu. \\ 4 Department of Mathematics, Shanghai Jiao Tong University, Shanghai 200030, Peo- \\ ple's Republic of China, e-mail: haoshen@sjtu.edu.cn.
}

\begin{abstract}
The intersection of two Steiner triple systems $(X, \mathcal{A})$ and $(X, \mathcal{B})$ is the set $\mathcal{A} \cap \mathcal{B}$. The fine intersection problem for Steiner triple systems is to determine for each $v$, the set $I(v)$, consisting of all possible pairs $(m, n)$ such that there exist two Steiner triple systems of order $v$ whose intersection $\mathcal{I}$ satisfies $\left|\cup_{A \in \mathcal{I}} A\right|=m$ and $|\mathcal{I}|=n$. We show that for $v \equiv 1$ or $3(\bmod 6),|I(v)|=\Theta\left(v^{3}\right)$, where previous results only imply that $|I(v)|=\Omega\left(v^{2}\right)$.
\end{abstract}

Key words. Steiner triple systems, intersection.

\section{Introduction}

For a set $X$ and non-negative integer $k$, denote by $\left(\begin{array}{l}X \\ k\end{array}\right)$ the set of all $k$-subsets of $X$. The support of $\mathcal{A} \subseteq 2^{X}$, denoted by $\operatorname{supp}(A)$, is the set $\cup_{A \in \mathcal{A}} A$. A set system is a pair $(X, \mathcal{A})$, where $X$ is a finite set of points, and $\mathcal{A} \subseteq 2^{X}$. The elements of $A$ are called blocks. The order of a set system is the number of points in the set system. Let $K$ be a set of positive integers. The set $K$ is a set of block sizes for $(X, \mathcal{A})$ if $|A| \in K$ for all $A \in \mathcal{A}$. A set system $(X, \mathcal{A})$ is said to be $k$-uniform if $\mathcal{A} \subseteq\left(\begin{array}{l}X \\ k\end{array}\right)$.

Let $(X, \mathcal{A})$ be a set system and let $\mathcal{G}=\left\{G_{1}, \ldots, G_{s}\right\}$ be a partition of $X$ into subsets called groups. The triple $(X, \mathcal{G}, \mathcal{A})$ is a group divisible design (GDD) when every 2 -subset of $X$ is either contained in exactly one block or in exactly one group. We denote a $\operatorname{GDD}(X, \mathcal{G}, \mathcal{A})$ by $K$-GDD if $K$ is a set of block sizes for $(X, \mathcal{A})$. The type of a $\operatorname{GDD}(X, \mathcal{G}, \mathcal{A})$ is the multiset $[|G|: G \in \mathcal{G}]$. When more convenient, we use the exponentiation notation to describe the type of a GDD: a GDD of type $g_{1}^{t_{1}} \cdots g_{s}^{t_{s}}$ is a GDD where there are exactly $t_{i}$ groups of size $g_{i}$, $1 \leq i \leq s$. 
A $\{3\}$-GDD of type $1^{v}$ is a Steiner triple system (STS) of order $v$, and is denoted $\operatorname{STS}(v)$. It is well-known that an $\operatorname{STS}(v)$ exists if and only if $v \equiv 1$ or 3 $(\bmod 6)($ see, for example, 3 ]). A partial triple system is a 3-uniform set system $(X, \mathcal{A})$ where every 2 -subset of $X$ is contained in at most one block of $\mathcal{A}$.

The intersection of two $K$-GDDs (of the same type) $\mathrm{D}_{1}=\left(X, \mathcal{G}, \mathcal{A}_{1}\right)$ and $\mathrm{D}_{2}=\left(X, \mathcal{G}, \mathcal{A}_{2}\right)$ is the set $\mathcal{I}\left(\mathrm{D}_{1}, \mathrm{D}_{2}\right)=\mathcal{A}_{1} \cap \mathcal{A}_{2} . \mathrm{D}_{1}$ and $\mathrm{D}_{2}$ are said to be disjoint if $\mathcal{I}\left(\mathrm{D}_{1}, \mathrm{D}_{2}\right)=\varnothing$. Let $f: 2^{\left(\begin{array}{l}X \\ 3\end{array}\right)} \rightarrow \Gamma$. The $f$-intersection problem for $K-G D D$ s of type $T$ is to determine the set

$$
\begin{aligned}
& \operatorname{Int}_{f}(K, T) \\
= & \left\{r \in \Gamma: \exists \text { two } K \text {-GDDs of type } T, \mathrm{D}_{1} \text { and } \mathrm{D}_{2} \text {, with } f\left(\mathcal{I}\left(\mathrm{D}_{1}, \mathrm{D}_{2}\right)\right)=r\right\} .
\end{aligned}
$$

The interest in this paper is the $f$-intersection problem for STS in the case when $f=\Phi$, where $\Phi: 2^{\left(\begin{array}{l}X \\ 3\end{array}\right)} \rightarrow \mathbb{Z}_{\geq 0}^{2}$ is defined as follows:

$$
\Phi(\mathcal{S})=(|\operatorname{supp}(\mathcal{S})|,|\mathcal{S}|), \text { for } \mathcal{S} \subseteq\left(\begin{array}{c}
X \\
3
\end{array}\right) .
$$

We call this the fine intersection problem for STS for the reason that both the number of blocks and the number of underlying points in the intersection are to be determined simultaneously.

All previous work on the intersection of STS can be cast in the context of $f$-intersection problems for STS, for appropriate choices of $f$.

Example 1. (Lindner and Rosa [6]) The classical problem of determining the possible number of blocks in the intersection of two $\operatorname{STS}(v)$ is equivalent to the $f$-intersection problem for STS with $f(\mathcal{S})=|\mathcal{S}|$.

Example 2. (Hoffman and Lindner [5]) The flower intersection problem for STS $(v)$ is equivalent to the $f$-intersection problem for STS, with

$f(\mathcal{S})= \begin{cases}|\mathcal{S}|, & \text { if } \mathcal{S} \text { contains a set of } \frac{v-1}{2} \text { blocks intersecting in a common point; } \\ \infty, & \text { otherwise. }\end{cases}$

Example 3. (Chee [2]) The disjoint intersection problem for STS is equivalent to the $f$-intersection problem for STS, with

$$
f(\mathcal{S})= \begin{cases}|\mathcal{S}|, & \text { if the blocks in } \mathcal{S} \text { are pairwise disjoint } \\ \infty, & \text { otherwise }\end{cases}
$$

This is also equivalent to determining the possible values of $n$ for which $(3 n, n) \in$ $\operatorname{Int}_{\Phi}\left(\{3\}, 1^{v}\right)$.

The purpose of this paper is to initiate the study on the fine intersection problem for STS. 


\section{Admissible Region}

In this section, we determine an admissible region for the fine intersection problem for STS; that is, we determine a subset of $\mathbb{Z}_{\geq 0}^{2}$ which contains $\operatorname{Int}_{\Phi}\left(\{3\}, 1^{v}\right)$.

A partial triple system $(X, \mathcal{A})$ of order $v$ is said to be maximum if for every partial triple system $(X, \mathcal{B})$ of order $v$, we have $|\mathcal{B}| \leq|\mathcal{A}|$. The number of blocks in a maximum partial triple system is denoted $D(2,3, v)$. For a vector $\mathbf{x}=\left(x_{1}, \ldots, x_{d}\right) \in \mathbb{Z}^{d}$, we denote by $\left.\mathbf{x}\right|_{i}, 1 \leq i \leq d$, the value $x_{i}$. For a set of vectors $S \subseteq \mathbb{Z}^{d}$, we denote by $\left.S\right|_{i}, 1 \leq i \leq d$, the projection of $S$ on the $i^{\text {th }}$ dimension: $\left.S\right|_{i}=\left\{\left.\mathbf{x}\right|_{i}: \mathbf{x} \in S\right\}$.

We state below some prior results that are useful in establishing an admissible region for $\operatorname{Int}_{\Phi}\left(\{3\}, 1^{v}\right)$.

Theorem 1. (Lindner and Rosa [6]) Let $b(v)=v(v-1) / 6$. Then for all $v \equiv 1$ or $3(\bmod 6), v \neq 9$, $\operatorname{Int}_{|\cdot|}\left(\{3\}, 1^{v}\right)=\{0, \ldots, b(v)\} \backslash\{b(v)-5, b(v)-3, b(v)-$ $2, b(v)-1\}$, and $\operatorname{Int}_{|\cdot|}\left(\{3\}, 1^{9}\right)=\{0,1,2,3,4,6,12\}$.

Theorem 2. (Doyen and Wilson [4]) Let $v, w \equiv 1$ or $3(\bmod 6)$, and $w<v$. There exists an $S T S(v)$ containing an $S T S(w)$ if and only if $v \geq 2 w+1$.

Lemma 1. Let $(m, n) \in \operatorname{Int}_{\Phi}\left(\{3\}, 1^{v}\right)$. Then the following conditions hold:

(i) $m / 3 \leq n \leq D(2,3, m)$;

(ii) $n \notin\{b(v)-5, b(v)-3, b(v)-2, b(v)-1\}$;

(iii) if $v=9$, then $n \notin\{5,8\}$;

(iv) $m \notin\{1,2,4\}$;

(v) if $n>b(v)-(v-1) / 2$, then $m=v$; and

(vi) if $m<v$ and $n=b(m)$, then $v \geq 2 m+1$.

Proof. To see that (i) holds, note that there exists a pair of $\operatorname{STS}(v)$ whose intersection is $\mathcal{I}$, such that $(\operatorname{supp}(\mathcal{I}), \mathcal{I})$ is a partial triple system of order $m$ having $n$ blocks. Hence, $n \leq D(2,3, m)$. That $m / 3 \leq n$ follows easily from the observation that the maximum number of points underlying $n$ blocks of size three is $3 n$.

Theorem 1 together with the observation that $\left.\operatorname{Int}_{\Phi}\left(\{3\}, 1^{v}\right)\right|_{2}=\operatorname{Int}_{|\cdot|}\left(\{3\}, 1^{v}\right)$ gives conditions (ii) and (iii).

The observation that $\left.\operatorname{Int}_{\Phi}\left(\{3\}, 1^{v}\right)\right|_{1}=\operatorname{Int}_{|\operatorname{supp}(\cdot)|}\left(\{3\}, 1^{v}\right)$ and that the support of the set of blocks of a partial triple system can never contain one, two, or four points gives condition (iv).

For condition (v), observe that each point of an $\operatorname{STS}(v)$ lies in $(v-1) / 2$ blocks. So unless we have less than $b(v)-(v-1) / 2$ blocks, we cannot drive the support down to less than $v$ points.

Any partial triple system of order $m$ and $n=b(m)$ blocks is an $\operatorname{STS}(m)$. Theorem 2 then implies that $v \geq 2 m+1$, from which condition (vi) follows.

We call the set

$$
\mathrm{A}(v)=\left\{(m, n) \in \mathbb{Z}_{\geq 0}^{2}:(m, n) \text { satisfies conditions (i)-(vi) of Lemma } 1\right\}
$$

the admissible region for $\operatorname{Int}_{\Phi}\left(\{3\}, 1^{v}\right)$. 
Lemma 2. Let $v \equiv 1$ or $3(\bmod 6)$. Then $\left|\operatorname{Int}_{\Phi}\left(\{3\}, 1^{v}\right)\right| \leq(1+o(1)) \frac{1}{18} v^{3}$.

Proof.

$$
\begin{aligned}
\mid \operatorname{Int}_{\Phi}\left(\{3\}, 1^{v}\right) & \leq|\mathrm{A}(v)| \\
& \leq \sum_{m=0}^{v}(D(2,3, m)-m / 3+1) \quad \text { (by condition (i) of Lemma 1) } \\
& =\sum_{m=0}^{v}\left(\frac{1}{6} m^{2}+o\left(m^{2}\right)\right) \\
& =(1+o(1)) \frac{1}{18} v^{3} .
\end{aligned}
$$

Existing results on the intersection of STS only determine that a negligible portion of $\mathrm{A}(v)$ belongs to $\operatorname{Int}_{\Phi}\left(\{3\}, 1^{v}\right)$. In particular, Theorem 1 only implies that $\left|\operatorname{Int}_{\Phi}\left(\{3\}, 1^{v}\right)\right| \geq(1+o(1)) \frac{1}{6} v^{2}$. The main result of this paper is the following.

Main Theorem For $v \equiv 1$ or $3(\bmod 6),\left|\operatorname{Int}_{\Phi}\left(\{3\}, 1^{v}\right)\right|=\Theta\left(v^{3}\right)$.

\section{Proof of the Main Theorem}

Our main tool is Wilson's Fundamental Construction for GDDs 9 .

\begin{tabular}{|ll|}
\hline \multirow{3}{*}{ Input: } & \multicolumn{1}{c|}{ Wilson's Fundamental Construction } \\
& (master) GDD $\mathrm{D}=(X, \mathcal{G}, \mathcal{A}) ;$ \\
& weight function $\omega: X \rightarrow \mathbb{Z}_{\geq 0} ;$ \\
& (ingredient) $K$-GDD $\mathrm{D}_{A}=\left(X_{A}, \mathcal{G}_{A}, \mathcal{B}_{A}\right)$ of type $[\omega(a): a \in A]$, \\
& for each block $A \in \mathcal{A}$, where \\
& $X_{A}=\cup_{a \in A}\{\{a\} \times\{1, \ldots, \omega(a)\}\}$ and \\
& $\mathcal{G}_{A}=\{\{a\} \times\{1, \ldots, \omega(a)\}: a \in A\}$. \\
Output: & $K$-GDD $\mathrm{D}^{*}=\left(X^{*}, \mathcal{G}^{*}, \mathcal{A}^{*}\right)$ of type $\left[\sum_{x \in G} \omega(x): G \in \mathcal{G}\right]$, where \\
& $X^{*}=\cup_{x \in X}(\{x\} \times\{1, \ldots, \omega(x)\})$, \\
& $\mathcal{G}^{*}=\left\{\cup_{x \in G}(\{x\} \times\{1, \ldots, \omega(x)\}): G \in \mathcal{G}\right\}$, and \\
Notation: & $\mathcal{A}^{*}=\cup_{A \in \mathcal{A}} \mathcal{B}_{A} \cdot \operatorname{WFC}\left(\mathrm{D}, \omega,\left\{\mathrm{D}_{A}: A \in \mathcal{A}\right\}\right)$. \\
Note: & By convention, for $x \in X,\{x\} \times\{1, \ldots, \omega(x)\}=\varnothing$ if $\omega(x)=0$. \\
\hline
\end{tabular}

The master GDDs we use are the class of $\{4\}$-GDDs of type $1^{u} t^{1}$, existence for which has been settled by Rees and Stinson [7].

Theorem 3. (Rees and Stinson [7]) There exists a $\{4\}-G D D$ of type $1^{u} t^{1}$ whenever $u \geq 2 t+1$ and

(i) $u \equiv 0$ or $3(\bmod 12)$ and $t \equiv 1$ or $7(\bmod 12)$; or 
(ii) $u \equiv 0$ or $9(\bmod 12)$ and $t \equiv 4$ or $10(\bmod 12)$.

We call the distinguished group of size $t$ in a $\{4\}$-GDD of type $1^{u} t^{1}$, the hole.

Lemma 3. The number of blocks in a $\{4\}-G D D$ of type $1^{u} t^{1}$ that are disjoint from the hole is $u(u-2 t-1) / 12$.

Proof. The number of blocks that have non-empty intersection with the hole is easily seen to be $u t / 3$. The total number of blocks in the GDD is $\left(\left(\begin{array}{c}u+t \\ 2\end{array}\right)-\left(\begin{array}{c}t \\ 2\end{array}\right)\right) / 6$. Hence, the number of blocks disjoint from the hole is $\left(\left(\begin{array}{c}u+t \\ 2\end{array}\right)-\left(\begin{array}{c}t \\ 2\end{array}\right)\right) / 6-u t / 3=$ $u(u-2 t-1) / 12$.

We also make use of the following result of Butler and Hoffman [1.

Theorem 4. (Butler and Hoffman [1]) Let $g$ and $t$ be positive integers such that $t \geq 3, g^{2}\left(\begin{array}{c}t \\ 2\end{array}\right) \equiv 0(\bmod 3)$, and $g(t-1) \equiv 0(\bmod 2)$. Let $b\left(g^{t}\right)=g^{2} t(t-1) / 6$ and denote by $I\left(g^{t}\right)=\left\{0, \ldots, b\left(g^{t}\right)\right\} \backslash\left\{b\left(g^{t}\right)-5, b\left(g^{t}\right)-3, b\left(g^{t}\right)-2, b\left(g^{t}\right)-1\right\}$. Then $\operatorname{Int}_{|\cdot|}\left(\{3\}, g^{t}\right)=I\left(g^{t}\right)$, except that

(i) $\operatorname{Int}_{|\cdot|}\left(\{3\}, 1^{9}\right)=I\left(1^{9}\right) \backslash\{5,8\}$;

(ii) $\operatorname{Int}_{|\cdot|}\left(\{3\}, 2^{4}\right)=I\left(2^{4}\right) \backslash\{1,4\}$;

(iii) $\operatorname{Int}_{|\cdot|}\left(\{3\}, 3^{3}\right)=I\left(3^{3}\right) \backslash\{1,2,5\}$; and

(iv) $\operatorname{Int}_{|\cdot|}\left(\{3\}, 4^{3}\right)=I\left(4^{3}\right) \backslash\{5,7,10\}$.

Let $\mathrm{D}=(X, \mathcal{G}, \mathcal{A})$ be a $\{4\}-\mathrm{GDD}$ of type $1^{u} t^{1}$, with $\mathcal{G}=\left\{G_{1}, \ldots, G_{u+1}\right\}$, where

$$
G_{i}= \begin{cases}\left\{x_{i}\right\}, & \text { if } 1 \leq i \leq u ; \text { and } \\ \left\{x_{u+1}, \ldots, x_{u+t}\right\}, & \text { if } i=u+1 .\end{cases}
$$

For $\alpha, \beta \geq 0$ such that $\alpha+\beta \leq t$, define the following weight function:

$$
\omega_{\alpha, \beta}(x)= \begin{cases}2, & \text { if } x \in\left\{x_{1}, \ldots, x_{u+\alpha}\right\} \\ 4, & \text { if } x \in\left\{x_{u+\alpha+1}, \ldots, x_{u+\alpha+\beta}\right\} ; \text { and } \\ 0, & \text { if } x \in\left\{x_{u+\alpha+\beta+1}, \ldots, x_{u+t}\right\} .\end{cases}
$$

We use Wilson's Fundamental Construction with D as master GDD and $\omega_{\alpha, \beta}$ as weight function. The required ingredient GDDs are $\{3\}$-GDDs of type $2^{4}$, type $2^{3} 4^{1}$, and type $2^{3}$. The following are results on the intersections of these ingredient GDDs.

Lemma 4. The following hold:

(i) $\operatorname{Int}_{|\cdot|}\left(\{3\}, 2^{3}\right)=\{0,4\}$;

(ii) $\operatorname{Int}_{|\cdot|}\left(\{3\}, 2^{4}\right)=\{0,2,8\}$; and

(iii) $0 \in \operatorname{Int}_{|\cdot|}\left(\{3\}, 2^{3} 4^{1}\right)$; 
Proof. (i) and (ii) follow from Theorem 4. The existence of a pair of disjoint $\{3\}$-GDDs of type $2^{3} 4^{1}$ is given in [8], proving (iii).

For a set $A$ of integers, we denote by $A+A$ the set $\{a+b: a, b \in A\}$, and denote by $\sum_{i=1}^{s} A$ the set $A+\cdots+A$ (s-fold sum).

Lemma 5. Let $s \geq 2$. Then $\left|\operatorname{Int}_{|\cdot|}\left(\{3\}, 2^{4}\right)\right|=4 s-2$.

Proof. We have $\sum_{i=1}^{s} \operatorname{Int}_{|\cdot|}\left(\{3\}, 2^{4}\right)=\{0,2,4, \ldots, 8 s\} \backslash\{8 s-10,8 s-4,8 s-2\}$.

Lemma 6. Let $m \geq 0$ and $r \in\{1,3,7,9,13,15,19,21\}$. Then for every $a \geq$ $\lceil(m+3) / 2\rceil$, there exists an integer $b \geq 0$ such that the following inequalities are all satisfied:

(i) $24(m-a)+r \leq 4(12 b+1)+1$;

(ii) $12 a \geq 2(12 b+1)+1$.

Proof. Let $b$ be the smallest integer such that inequality (i) holds. Then

$$
b=\left\lceil\frac{24(m-a)+r-5}{48}\right\rceil \geq\left\lceil\frac{r-5}{48}\right\rceil \geq\left\lceil-\frac{4}{48}\right\rceil=0 .
$$

It remains to show that inequality (ii) holds. To see that this is the case, observe that

$$
\begin{aligned}
2(12 b+1)+1 & =24 b+3 \\
& =24\left\lceil\frac{24(m-1)+r-5}{48}\right\rceil+3 \\
& =24\left\lceil\frac{m-a}{2}+\frac{r-5}{48}\right\rceil+3 \\
& \leq 24\left\lceil\frac{m-a}{2}+\frac{16}{48}\right\rceil+3 \\
& \leq 24\left(\frac{m-a}{2}+1\right)+3 \\
& =12(m-a)+27 \\
& \leq 12(2 a-3-a)+27 \\
& =12 a-9 \\
& \leq 12 a .
\end{aligned}
$$

Let $m \geq 0$ and $r \in\{1,3,7,9,13,15,19,21\}$. We now construct a pair of $\operatorname{STS}(24 m+r)$ via Wilson's Fundamental Construction. Write $24 m+r$ as $24 a+$ $24(m-1)+r$, where $a \geq\lceil(m+3) / 2\rceil$. Choose $b$ to be the smallest non-negative integer so that the inequalities $24(m-a)+r \leq 4(12 b+1)+1$ and $12 a \geq$ 
$2(12 b+1)+1$ are both satisfied. Such a $b$ exists by Lemma 6. Choose also nonnegative integers $\alpha$ and $\beta$ such that $0 \leq \alpha+\beta \leq 12 b+1$ and $2 \alpha+4 \beta+1=$ $24(m-a)+r$. This is always possible because $\{2 \alpha+4 \beta+1: 0 \leq \alpha+\beta \leq$ $12 b+1\}=\{1,3,5, \ldots, 4(12 b+1)+1\} \ni 24(m-a)+r$.

Now, take $\mathrm{D}=\{X, \mathcal{G}, \mathcal{A})$ to be a $\{4\}$-GDD of type $1^{12 a}(12 b+1)^{1}$. The existence of such a GDD is implied by the inequality $12 a \geq 2(12 b+1)+1$ (via Theorem 3). Let $\mathrm{G}=\mathrm{WFC}\left(\mathrm{D}, \omega_{\alpha, \beta},\left\{\mathrm{D}_{A}: A \in \mathcal{A}\right\}\right)$ and $\mathrm{G}^{\prime}=\mathrm{WFC}\left(\mathrm{D}, \omega_{\alpha, \beta},\left\{\mathrm{D}_{A}^{\prime}: A \in\right.\right.$ $\mathcal{A}\}$ ), where $\mathrm{D}_{A}$ and $\mathrm{D}_{A}^{\prime}$ are a pair of

(i) $\{3\}$-GDDs of type $2^{4}$ intersecting in $\mu_{A}$ blocks, if $A$ is disjoint from the hole of $\mathrm{D}$;

(ii) disjoint $\{3\}$-GDDs of type $2^{4}$, if $A$ contains a point of weight two from the hole of $\mathrm{D}$;

(iii) disjoint $\{3\}$-GDDs of type $2^{3} 4^{1}$, if $A$ contains a point of weight four from the hole of $\mathrm{D}$; and

(iv) disjoint $\{3\}$-GDDs of type $2^{3}$, if $A$ contains a point of weight zero from the hole of D.

Such ingredient GDDs all exist by Lemma 4 .

It is clear from the description of Wilson's Fundamental Construction that G and $\mathrm{G}^{\prime}$ are two $\{3\}$-GDDs of type $2^{12 a}(2 \alpha+4 \beta)^{1}$, where $0 \leq \alpha+\beta \leq 12 b+1$, intersecting in $\sum_{A \in \mathcal{A}} \mu_{A}$ blocks, whose support is disjoint from the group of size $2 \alpha+4 \beta$. Now add a point to each of $\mathrm{G}$ and $\mathrm{G}^{\prime}$ to obtain $\{3,2 \alpha+4 \beta+1\}$-GDDs of type $1^{24 a+2 \alpha+4 \beta+1}$ with exactly one block of size $2 \alpha+4 \beta+1$. Replace the block of size $2 \alpha+4 \beta+1=24(m-a)+r$ in each of these GDDs with the respective blocks from a pair of disjoint $\operatorname{STS}(24(m-a)+r)$ (which exists by Theorem 1). The result is a pair of $\operatorname{STS}(24 m+r)$ intersecting in $12 a+\sum_{A \in \mathcal{A}} \mu_{A}$ blocks whose support contains exactly $24 a+1$ points.

By varying $a$ and $\mu_{A}$, this shows that

$$
\left|\operatorname{Int}_{\Phi}\left(\{3\}, 1^{24 m+r}\right)\right| \geq \sum_{a=\lceil(m+3) / 2\rceil}^{m}\left|\sum_{i=1}^{a(12 a-2(12 b+1)-1)} \operatorname{Int}_{|\cdot|}\left(\{3\}, 2^{4}\right)\right|
$$

Lemma 7. For $a \geq\lceil(m+3) / 2\rceil$, we have $12 a-2(12 b+1)-1 \geq 24 a-12 m-27$.

Proof. By the proof of Lemma 6, we know that $b \leq\left\lceil\frac{24(m-a)+r-5}{48}\right\rceil \leq 1+(m-$ a) $/ 2$. So,

$$
\begin{aligned}
12 a-2(12 b+1)-1 & =12 a-24 b-3 \\
& \geq 12 a-24(1+(m-a) / 2)-3 \\
& =24 a-12 m-27 .
\end{aligned}
$$


By Lemma [7, inequality (1) implies

$$
\begin{aligned}
\left|\operatorname{Int}_{\Phi}\left(\{3\}, 1^{24 m+r}\right)\right| \geq & \sum_{a=\lceil(m+3) / 2\rceil}^{m}\left|\sum_{i=1}^{a(24 a-12 m-27)} \operatorname{Int}_{|\cdot|}\left(\{3\}, 2^{4}\right)\right| \\
& =\sum_{a=\lceil(m+3) / 2\rceil}^{m}(4 a(24 a-12 m-27)-2) \quad \text { (via Lemma 5]) } \\
& =\left(96 \sum_{a=\lceil(m+3) / 2\rceil}^{m} a^{2}\right)-\left(4(12 m+27) \sum_{a=\lceil(m+3) / 2\rceil}^{m} a\right)- \\
& \left(\sum_{a=\lceil(m+3) / 2\rceil}^{m} 2\right) \\
\geq & \left(28 m^{3}+o\left(m^{3}\right)\right)-\left(18 m^{3}+o\left(m^{3}\right)\right)-o\left(m^{3}\right) \\
& =10 m^{3}+o\left(m^{3}\right) .
\end{aligned}
$$

This shows that for $v \equiv 1$ or $3(\bmod 6)$,

$$
\left|\operatorname{Int}_{\Phi}\left(\{3\}, 1^{v}\right)\right| \geq(1+o(1)) \frac{5}{6912} v^{3},
$$

which together with Lemma 2 proves the Main Theorem.

\section{Conclusion}

In this paper, we initiated the study on the fine intersection problem for STS. We established that $\left|\operatorname{Int}_{\Phi}\left(\{3\}, 1^{v}\right)\right|=\Theta\left(v^{3}\right)$ for $v \equiv 1$ or $3(\bmod 6)$. There remain many interesting unsolved problems:

(i) What is the exact asymptotics of $\left|\operatorname{Int}_{\Phi}\left(\{3\}, 1^{v}\right)\right|$ ? There remains a wide gap between our lower and upper bounds on $\left|\operatorname{Int}_{\Phi}\left(\{3\}, 1^{v}\right)\right|$. We think the upper bound is probably the truth and make the conjecture that $\left|\operatorname{Int}_{\Phi}\left(\{3\}, 1^{v}\right)\right|=$ $(1+o(1)) \frac{1}{18} v^{3}$.

(ii) Determine completely the set $\operatorname{Int}_{\Phi}\left(\{3\}, 1^{v}\right)$. This problem is probably very difficult.

(iii) What is the number of non-isomorphic partial triple systems that can underly the intersection of two $\operatorname{STS}(v)$ ?

(iv) Determine all non-isomorphic partial triple systems that can underly the intersection of two $\operatorname{STS}(v)$.

Intersection problems for STS remain well alive three decades after the seminal paper of Lindner and Rosa [6], 


\section{References}

1. R. A. R. Butler and D. G. Hoffman. Intersections of group divisible triple systems. Ars Combin. 34, (1992) 268-288.

2. Y. M. Chee. Steiner triple systems intersecting in pairwise disjoint blocks. Electron. J. Combin. 11, (2004) Research Paper 27, 17 pp. (electronic).

3. C. J. Colbourn and A. Rosa. Triple Systems. Oxford Mathematical Monographs, The Clarendon Press Oxford University Press, New York, 1999.

4. J. Doyen and R. M. Wilson. Embeddings of Steiner triple systems. Discrete Math. 5, (1973) 229-239.

5. D. G. Hoffman and C. C. Lindner. The flower intersection problem for Steiner triple systems. Combinatorial Design Theory. vol. 149 of North-Holland Math. Stud., North-Holland, Amsterdam, 1987, pp. 243-248.

6. C. C. Lindner and A. Rosa. Steiner triple systems having a prescribed number of triples in common. Canad. J. Math. 27, (1975) 1166-1175.

7. R. Rees and D. R. Stinson. On the existence of incomplete designs of block size four having one hole. Utilitas Math. 35, (1989) 119-152.

8. P. J. Schellenberg and D. R. Stinson. Threshold schemes from combinatorial designs. J. Combin. Math. Combin. Comput. 5, (1989) 143-160.

9. R. M. Wilson. An existence theory for pairwise balanced designs I - Composition theorems and morphisms. J. Combin. Theory Ser. A 13, (1972) 220-245. 